\title{
Armas metálicas en la «Motilla» de Santa María del Retamar (Argamasilla de Alba, Ciudad Real)
}

\author{
A. Hernando Grande * \\ C. Galan Saulnier**
}

La presencia de armas de metal en los yacimientos protohistóricos puede ser interpretada, y de hecho lo ha sido, desde distintos puntos de vista, y en consecuencia de distintas formas.

En el caso concreto de la Edad del Bronce de la Península Ibérica, la existencia de armas en los asentamientos y, especialmente, en los ajuares funerarios ha llevado a un buen número de investigadores, cuyos nombres y trabajos seria muy largo citar aqui en su totalidad, a proponer diferentes interpretaciones y formular diversas hipótesis relativas al significado socio-cultural de la misma, interpretaciones e hipótesis de trabajo que no es nuestro propósito analizar aquí y ahora, pues excedería los limites de este trabajo.

Asimismo, aun dejando de momento al margen el problema de la identificación del sentido o significado socioculturales de las armas de metal en los yacimientos de nuestra Protohistoria en que aparecen, no podemos obviar sin embargo el hecho de que su presencia es un buen indicador de algo que indudablemente influyó de forma decisiva no sólo en el desarrollo, sino también en la caracterización cultural de las pobla-

* Departamento de Prehistoria e Historia Antigua de la UNED.

** Universidad Autonoma de Madrid. 
ciones peninsulares del $|l|$ y el II milenios a. C.: su grado de desarrollo económico ${ }^{1}$.

De cualquier forma, pensamos que hay una realidad que es necesario tener en cuenta a la hora de interpretar la presencia de armas metálicas, y es el hecho de que dicha interpretación tal vez no deba ser la misma si se trata de armas procedentes de un área de ocupación que si proceden de ajuares funerarios, pues no podemos olvidar que en los ajuares funerarios nos encontramos con lo que quienes lo depositaron quisieron dejar "para la posteridad", mientras que en los asentamientos hallamos simplemente los restos de su ocupacion, es decir, lo que por una $u$ otra razón quedó alli y, a través de los años, ha llegado hasta nosotros.

En una palabra, estos restos arqueológicos, como cualquier otro, no deben ser interpretados individualizadamente, es decir, aislándolos de su contexto, sino teniendo en cuenta su situación en el propio yacimiento, y entendiendo por "contexto" no sólo el conjunto de materias y estructuras asociadas a ellos, sino también todas las circunstancias que los rodean, tales como su localización exacta y las características topográficas, morfológicas, etc. del lugar, y por supuesto, en el caso que nos ocupa, todo ello sin olvidar que algunas armas, como las puntas de flecha y los puñales, ya plantean por si mismas serios problemas derivados de sus propias caracteristicas, como es el de la identificación de su verdadera funcionalidad, lo que dificulta la interpretación de su presencia; no podemos pasar por alto el hecho de que las primeras pudieron ser destinadas a la práctica de actividades tanto bélicas como cinegéticas, mientras que los segundos, denominados genéricamente "puñales", pudieron ser armas sensu stricto, pero pudieron ser también artefactos de uso doméstico o industrial, es decir, pudieron haber sido utilizados como puñales o como cuchillos, o bien como ambas cosas al tiempo y según las necesidades del momento.

Es evidente pues que tanto al intentar aproximarnos al conocimiento del papel que representaron las armas metálicas en un complejo cultural, como al intentar identificar si su presencia en un yacimiento concreto es indicativa del nivel tecnológico y/o económico de quienes dejaron en él huellas de su paso por la Historia, o si esa presencia representa una

Galan Saulnier, C., Poyato holgado, C. y Sanchez Meseguer, J.: "Aplicaciones de Inteligencia Artificial y "Sistemas Expertos" a la Arqueologia. Un 'Experto' en economia del IIl milenio de la Península Ibérica", Cuadernos de Prehistoria y Arqueologia de la UAM (en prensa). 
sociedad en la que la posesión de objetos metálicos suponia la posesión de unos elementos de prestigio, en cualquiera de esas situaciones habremos de plantearnos interrogantes tales como:

¿Cómo son esas armas?

¿Dónde y cómo estaban en el yacimiento?

¿Qué contexto las acompaña?

¿En qué circunstancias aparecen esas armas en otros yacimientos del mismo Complejo Cultural?

En las líneas siguientes trataremos de dar respuesta a esas preguntas ante un caso concreto, para finalmente exponer algo que, en el estado actual de nuestras investigaciones, de momento sólo podemos plantear a modo de hipótesis: el sentido de la presencia de las armas metálicas recuperadas hasta el momento en nuestro yacimiento manchego.

MORFOLOGÍA DE LAS ARMAS DE METAL DE LA MOTILLA DE SANTA MARIA DEL RETAMAR

\section{CARACTERISTICAS}

\section{Puntas de Flecha}

Pieza núm. 1: núm. inv. MR-84-0064 (fig. 1, 1)

long. máx.: $70 \mathrm{~mm}$

anch. máx.: $12 \mathrm{~mm}$

grosor medio de la hoja: $3 \mathrm{~mm}$

grosor medio del pedúnculo: $4 \mathrm{~mm}$

long. de la hoja: $40 \mathrm{~mm}$

long. del pedúnculo: $30 \mathrm{~mm}$

Descripción: 

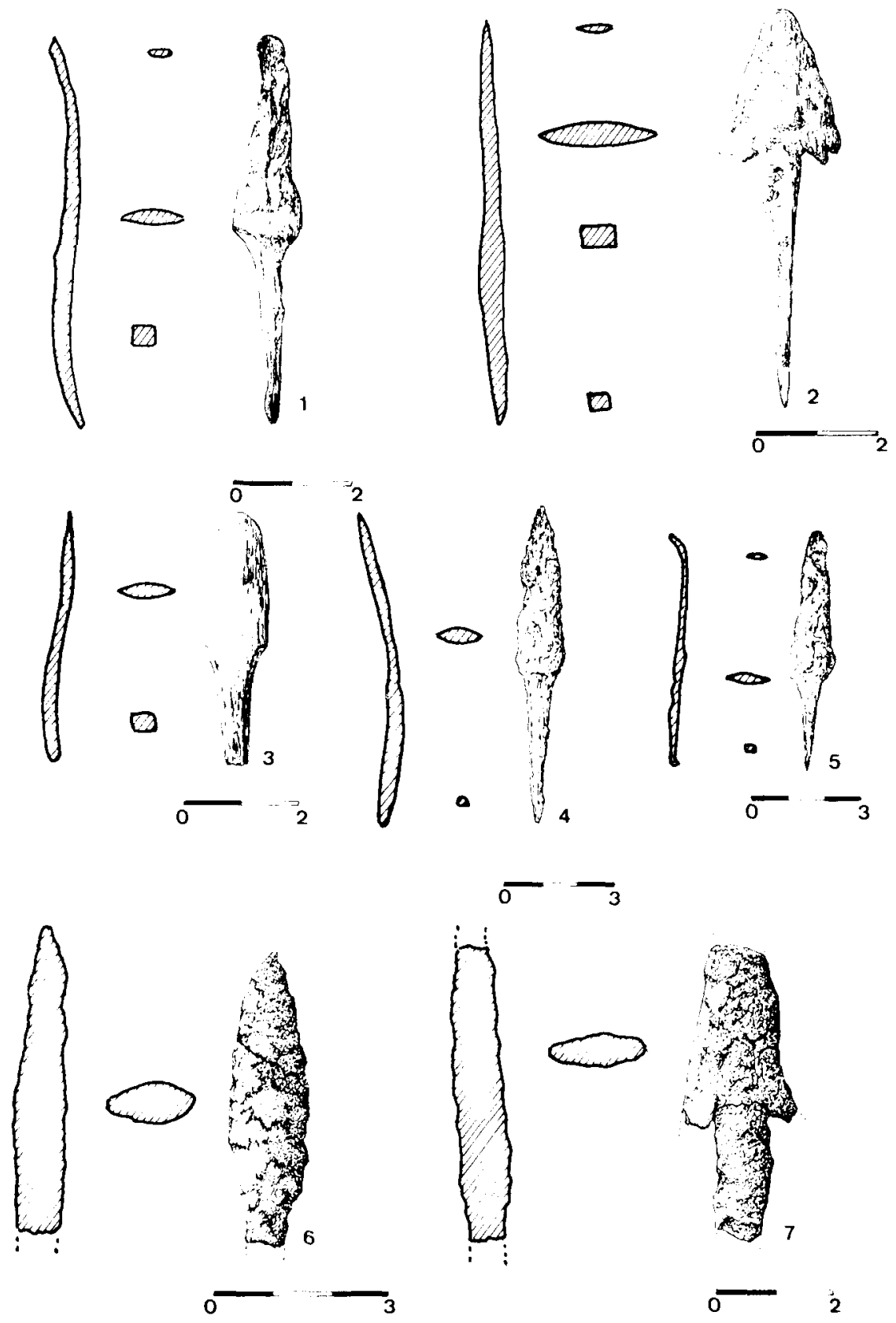

Fig. 1. 
Punta de flecha completa, de hoja triangular de sección lenticular, con pedúnculo corto de sección cuadrangular. En la intersección con el pedúnculo, la hoja disminuye su anchura presentando dos entrantes que se corresponden con las tradicionalmente conocidas como "aletas iniciadas». La sinuosa sección longitudinal de la pieza denota la deformación de la misma, debido muy posiblemente al choque con una superficie dura.

Pieza núm. 2: núm. inv. MR-84-0534 (fig. 1, 2)

long. máx.: $68 \mathrm{~mm}$

anch. máx.: $21 \mathrm{~mm}$

grosor medio de la hoja: $4 \mathrm{~mm}$

grosor medio del pedúnculo: $4 \mathrm{~mm}$

long. de la hoja: $24 \mathrm{~mm}$

long. del pedúnculo: $44 \mathrm{~mm}$

peso: $5.608 \mathrm{~g}$

\section{Descripción:}

Punta de flecha de hoja triangular de sección lenticular, con pedúnculo muy largo de sección cuadrangular y aletas laterales pronunciadas que se conservan fragmentadas e incompletas; la pieza presenta su sección longitudinal recta.

Pieza núm. 3: núm. inv. MR-84-0652 (fig. 1, 3)

long. máx: $43 \mathrm{~mm}$

anch. máx.: $11 \mathrm{~mm}$

grosor medio de la hoja: $2 \mathrm{~mm}$

grosor medio del pedúnculo: $3 \mathrm{~mm}$

long. de la hoja: $25 \mathrm{~mm}$

long. del pedúnculo: $18 \mathrm{~mm}$

peso: $3.900 \mathrm{~g}$

materia: cobre

composición: $\quad \mathrm{Fe}(0.072 \%), \quad \mathrm{Ni}(0.122 \%), \quad \mathrm{Cu}(98.91 \%), \quad \mathrm{Zn}$ (ind.), $\operatorname{As}(0.337 \%), \operatorname{Ag}(0.013 \%), \operatorname{Sn}(0.006 \%), \operatorname{Sb}(0.016 \%), \mathrm{Pb}(0.154 \%)$. 


\section{Descripción:}

Punta de flecha completa con hoja de forma elipsoide con los bordes prácticamente paralelos y la punta muy redondeada, de sección lenticular; presenta un pedúnculo corto de sección cuadrangular en cuya intersección con la hoja se aprecian dos escotaduras, por lo que, como la pieza núm. 1, responde al tipo tradicional denominado "de aletas incipientes". Por lo que respecta a su sección longitudinal, la parte correspondiente a la hoja se encuentra curvada también muy posiblemente como consecuencia de su lanzamiento contra una superficie dura.

Pieza núm. 4: núm. inv. MR-84-1104 (fig. 1, 4)

long. máx:: $85 \mathrm{~mm}$

anch. máx.: $13 \mathrm{~mm}$

grosor medio de la hoja: $4 \mathrm{~mm}$

grosor medio del pedúnculo: $3 \mathrm{~mm}$

long. de la hoja: $45 \mathrm{~mm}$

long. del pedúnculo: $40 \mathrm{~mm}$

peso: $8.770 \mathrm{~g}$

materia: cobre

composición: $\mathrm{Fe}(0.040 \%), \mathrm{Ni}(0.027 \%), \mathrm{Cu}(98.88 \%), \quad \mathrm{Zn}(0.186 \%)$, $\operatorname{As}(0.228 \%), \operatorname{Ag}(0.006 \%), \operatorname{Sn}(0.009 \%), \mathrm{Sb}(0.012 \%), \mathrm{Pb}$ (ind.).

Descripción:

Punta de flecha de hoja triangular de sección lenticular, con pedúnculo largo de sección circular. Como en otras piezas, la hoja se ensancha ligeramente en su unión con el pedúnculo, por lo que corresponderia también al tipo tradicional de "aletas iniciadas". Asimismo, la sección longitudinal muestra como la parte de la hoja esta curvada posiblemente por la causa ya citada.

Pieza núm. 5: núm. inv. MR-84-1105 (fig. 1, 5)

long. máx.: $68 \mathrm{~mm}$

anch. máx.: $12 \mathrm{~mm}$ 
grosor medio de la hoja: $2.5 \mathrm{~mm}$

grosor medio del pedúnculo: $2 \mathrm{~mm}$

long. de la hoja: $43 \mathrm{~mm}$

long. del pedúnculo: $25 \mathrm{~mm}$

peso: $4.267 \mathrm{~g}$

materia: cobre

composición: $\mathrm{Fe}(0.144 \%), \quad \mathrm{Ni}(0.066 \%), \quad \mathrm{Cu}(99.17 \%), \quad \mathrm{Zn}(\mathrm{ind}$.$) ,$ $\operatorname{As}(0.354 \%), \operatorname{Ag}(0.007 \%), \operatorname{Sn}(0.013 \%), \operatorname{Sb}(0.004 \%), \mathrm{Pb}($ ind.).

Descripción:

Punta de flecha de hoja triangular con sección lenticular, con pedúnculo corto de sección cuadrangular y extremo muy afilado. El ensanche de la hoja en su unión con el pedúnculo da lugar a "aletas iniciadas" del tipo de las anteriores. La pieza está completa pero su sección longitudinal muestra la punta de la hoja totalmente doblada.

Pieza núm. 6: núm. inv. MR-86-5230 (fig. 1, 6)

long. máx. de lo conservado: $49 \mathrm{~mm}$

anch. máx. de lo conservado: $16 \mathrm{~mm}$

grosor medio: $7 \mathrm{~mm}$

peso: $7.244 \mathrm{~g}$

material: cobre

composición: $\quad \mathrm{Fe}(0.203 \%), \quad \mathrm{Ni}(0.111 \%), \quad \mathrm{Cu}(98.46 \%) \quad \mathrm{Zn}$ (ind.), $\mathrm{As}(0.874 \%), \mathrm{Ag}(0.004 \%)$, Sn(ind.), $\mathrm{Sb}(0.014 \%), \mathrm{Pb}$ (ind.).

Descripción:

Punta de flecha incompleta, de hoja foliforme cuya sección aparece ovalada debido a la corrosión que ha sufrido la pieza; la sección longitudinal de la parte conservada es recta, no quedando restos del pedúnculo que con seguridad tuvo.

Pieza núm. 7: núm. inv. MR-86-5273 (fig. 1, 7)

long. máx. de lo conservado: $50 \mathrm{~mm}$

anch. máx. de lo conservado: $20 \mathrm{~mm}$ 
grosor medio de la hoja: $5 \mathrm{~mm}$

grosor medio del pedúnculo: $9 \mathrm{~mm}$

peso: $7.954 \mathrm{~g}$

materia: cobre

composición: $\quad \mathrm{Fe}(0.234 \%), \quad \mathrm{Ni}(0.100 \%), \quad \mathrm{Cu}(97.19 \%), \quad \mathrm{Zn}$ (ind.), $\operatorname{As}(1.591 \%), \operatorname{Ag}(0.006 \%)$, Sn(ind.), Sb(0.026\%), $\mathrm{Pb}($ ind.).

\section{Descripción:}

Punta de flecha de hoja triangular de sección ovalada debido también a su estado de conservación. Presenta aletas laterales pronunciadas y pedúnculo muy grueso, debido también a la corrosión, hallándose unas y otro fragmentados y por tanto la pieza incompleta. La sección longitudinal de la pieza es recta.

Pieza núm. 8: núm. inv. MR-87-6321 (fig. 2, 8)

long. máx.: $78 \mathrm{~mm}$

anch. $\max : 11 \mathrm{~mm}$

grosor medio de la hoja: $4 \mathrm{~mm}$

grosor medio del pedúnculo: $4 \mathrm{~mm}$

long. de la hoja: $45 \mathrm{~mm}$

long. del pedúnculo: $34 \mathrm{~mm}$

peso: $6.913 \mathrm{~g}$

materia: cobre

Composición: $\mathrm{Fe}(0.076 \%), \quad \mathrm{Ni}(0.071 \%), \quad \mathrm{Cu}(97.28 \%), \quad \mathrm{Zn}$ (ind.), $\operatorname{As}(1.492 \%), \operatorname{Ag}(0,001 \%), \operatorname{Sn}(0.018 \%), \operatorname{Sb}(0.011 \%), \mathrm{Pb}($ ind.)

Descripción:

Punta de flecha de hoja triangular de sección lenticular, con pedúnculo corto de sección cuadrangular; en la zona de arranque del pedúnculo presenta un pequeño apéndice lateral a modo de espolón. La sección longitudinal de la pieza, que se halla fragmentada e incompleta, pues el espolón ha desaparecido con posterioridad a su extracción debido a su avanzado estado de corrosión, es recta. 

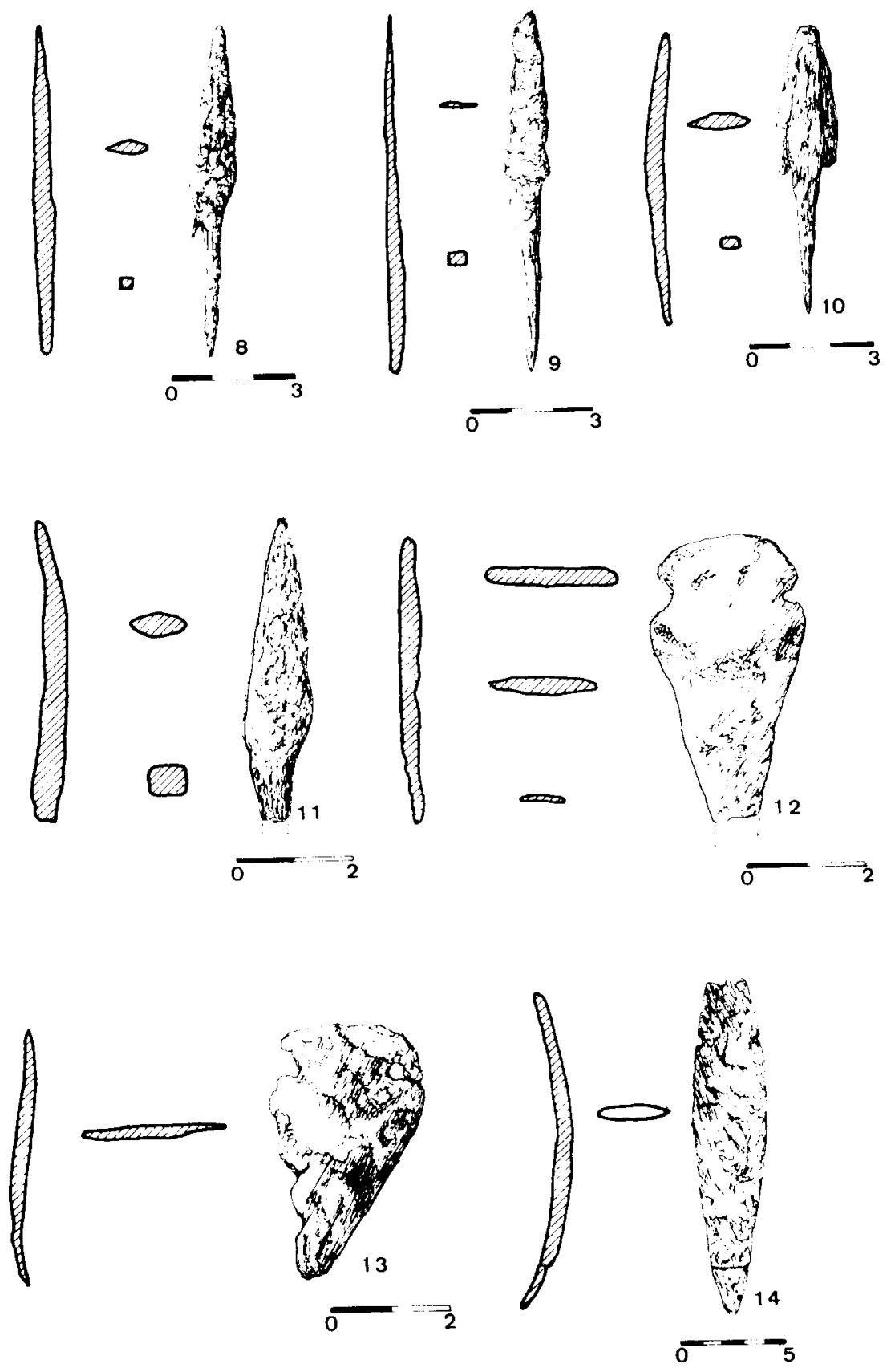

Fig. 2. 
Pieza núm. 9: núm. inv. MR-87-6332 (fig. 2, 9)

long. máx.: $85 \mathrm{~mm}$

anch. máx.: $11 \mathrm{~mm}$

grosor medio de la hoja: $2 \mathrm{~mm}$

grosor medio del pedúnculo: $4 \mathrm{~mm}$

long. de la hoja: $40 \mathrm{~mm}$

long. del pedúnculo: $45 \mathrm{~mm}$

peso: $8.193 \mathrm{~g}$

materia: cobre

composición: $\quad \mathrm{Fe}(0.068 \%), \quad \mathrm{Ni}(0.062 \%), \quad \mathrm{Cu}(95.37 \%), \quad \mathrm{Zn}$ (ind.), $\mathrm{As}(3.558 \%), \mathrm{Ag}$ (ind.), $\mathrm{Sn}$ (ind.), $\mathrm{Sb}(0.016 \%), \mathrm{Pb}$ (ind.).

Descripción:

Punta de flecha que se halló fragmentada e incompleta, asi como muy deteriorada, lo que impide apreciar con exactitud la forma de la hoja, que parece triangular y de sección lenticular; su pedúnculo es largo y de sección cuadrangular y recta la sección longitudinal de la pieza.

Pieza núm. 10: núm. inv. MR-87-6336 (fig. 2, 10)

long. máx.: $68 \mathrm{~mm}$

anch. máx.: $15 \mathrm{~mm}$

grosor medio de la hoja: $4.2 \mathrm{~mm}$

grosor medio del pedúnculo: $2.8 \mathrm{~mm}$

long. de la hoja: $33 \mathrm{~mm}$

long. del pedúnculo: $35 \mathrm{~mm}$

peso: $5.807 \mathrm{~g}$

materia: cobre

composición: $\quad \mathrm{Fe}(0.200 \%), \quad \mathrm{Ni}($ ind. $), \quad \mathrm{Cu}(97.57 \%), \quad \mathrm{Zn}(0.232 \%)$, As(1.864\%), Ag(ind.), Sn(ind.), Sb(0.047\%), $\mathrm{Pb}(\mathrm{tr})$.

Descripción:

Punta de flecha de hoja triangular suavemente biselada en sus bordes, lo que hace que su sección lenticular se encuentre aplanada en la parte superior. Presenta aletas laterales marcadas, pedúnculo largo de sección cuadrangular y sección longitudinal ligeramente curvada en la 
parte correspondiente a la hoja. La pieza está fragmentada e incompleta, no conservandose las aletas completas.

Pieza núm. 11: núm. inv. MR-87-6341 (fig. 2, 11)

long. máx. de lo conservado: $51 \mathrm{~mm}$

anch. máx.: $12 \mathrm{~mm}$

grosor medio de la hoja: $4 \mathrm{~mm}$

grosor medio del pedúnculo: $5 \mathrm{~mm}$

long. de la hoja: $43 \mathrm{~mm}$

long. del pedúnculo conservada: $8 \mathrm{~mm}$

peso: $4.893 \mathrm{~g}$

materia: cobre

composición: $\mathrm{Fe}(0.211 \%), \quad \mathrm{Ni}(0.055 \%), \quad \mathrm{Cu}(97.37 \%), \quad \mathrm{Zn}$ (ind.), $\operatorname{As}(2.129 \%), \operatorname{Ag}(0.027 \%), \operatorname{Sn}(0.010 \%), \mathrm{Sb}(0.030 \%), \mathrm{Pb}$ (ind.)

Descripción:

Punta de flecha de hoja triangular de sección prácticamente ovalada debido a la corrosión, con un pedúnculo de sección cuadrangular del que solo se conserva el arranque; en la intersección del pedúnculo y la hoja, ésta se ensancha por lo que responde como otras piezas al tipo tradicional de "aletas incipientes". La pieza está pues fragmentada e incompleta y su sección longitudinal muestra una ligera curvatura.

\section{Puñales}

Pieza núm. 12: núm. inv. MR-84-0093 (fig. 2, 12)

long. máx. conservada: $49 \mathrm{~mm}$

anch. máx.: $27 \mathrm{~mm}$

grosor medio de la hoja: $3 \mathrm{~mm}$

Descripción: 
Hoja de puñal de remaches, plana y triangular, que presenta la zona de la empuñadura redondeada y con dos escotaduras laterales que bien pudieran ser dos orificios para sendos remaches cuyos bordes se han roto. La pieza se encuentra fragmentada e incompleta, faltando la punta de la hoja, aunque su sección longitudinal denota que se conserva recta.

Pieza núm. 13: núm. inv. MR-85-3506 (fig. 2, 13)

long. máx.: $44 \mathrm{~mm}$

anch. máx.: $27 \mathrm{~mm}$

grosor medio: $2 \mathrm{~mm}$

peso: $7.238 \mathrm{~g}$

materia: cobre

composición: $\quad \mathrm{Fe}(0.010 \%), \quad \mathrm{Ni}(0.037 \%), \quad \mathrm{Cu}(98.97 \%), \quad \mathrm{Zn}$ (ind.), $\operatorname{As}(0.345 \%), \operatorname{Ag}(0.007 \%)$, Sn(ind.), $\mathrm{Sb}(0.003), \mathrm{Pb}$ (ind.).

Descripción:

Hoja de puñal de remaches triangular y plana, de características morfológicas semejantes al anterior; presenta en la zona de la empuñadura dos orificios laterales de los cuales uno se encuentra fragmentado, de lo que se desprende que los dos remaches correspondientes se hallaban dispuestos horizontalmente. La sección longitudinal presenta una curvatura sinuosa y uno de los bordes de la pieza, que está incompleta al faltar un pequeño fragmento de la empuñadura, se halla también deformado.

Pieza núm. 14: núm. inv. MR-86-5243 (fig. 2, 14)

log. máx.: $155 \mathrm{~mm}$

anch. máx.: $25 \mathrm{~mm}$

grosor medio: $6.5 \mathrm{~mm}$

peso: $90.630 \mathrm{~g}$

materia: cobre

composición: $\quad \mathrm{Fe}(0.134 \%), \quad \mathrm{Ni}(0.095 \%), \quad \mathrm{Cu}(96.28 \%), \quad \mathrm{Zn}($ ind.), $\operatorname{As}(3.390 \%), \operatorname{Ag}($ ind.), $\mathrm{Sn}(0.006 \%), \mathrm{Sb}(0.028 \%), \mathrm{Pb}$ (ind.). 


\section{Descripción:}

Hoja de puñal, de remaches, plana y ojival pues sus bordes son ligeramente cóncavos; la parte correspondiente a la empuñadura presenta un perfil prácticamente cuadrangular, con las esquinas redondeadas, y en ella se hallan tres orificios para los remaches dispuestos en triángulo, de forma que se hallan dos en los bordes laterales y el tercero en la parte central. La pieza se encuentra fragmentada pero completa, y su sección longitudinal denota una ligera curvatura.

\section{ANÁLISIS}

\section{Punta de flecha}

\section{A. Morfologia}

El análisis morfológico de las piezas que estudiamos se ha realizado partiendo de la diferenciación en ellas de sus tres partes fundamentales, distal, mesial y proximal, y el consiguiente análisis de las características de las mismas, tal como queda gráficamente expuesto en las tablas números 1 y 2 , a la vista de las cuales es fácil deducir las características del conjunto, como ahora expondremos.

Por 10 que respecta a las puntas de flecha (tabla 1), es evidente que se trata en todos los casos de puntas pedúnculadas, pero también lo es que su morfología no es uniforme, pudiéndiendose observar lo siguiente:

a) Extremo distal

En consecuencia con la forma de las hojas, hay un claro predominio de las puntas con el extremo distal de lados rectos convergentes, es decir, con su punta angulosa - puntas de hoja triangular- mientras que un solo caso, que se corresponde con una hoja foliforme, presenta un pertil ojival, y otro, correspondiente a una hoja de bordes paralelos, tiene la punta redondeada. 

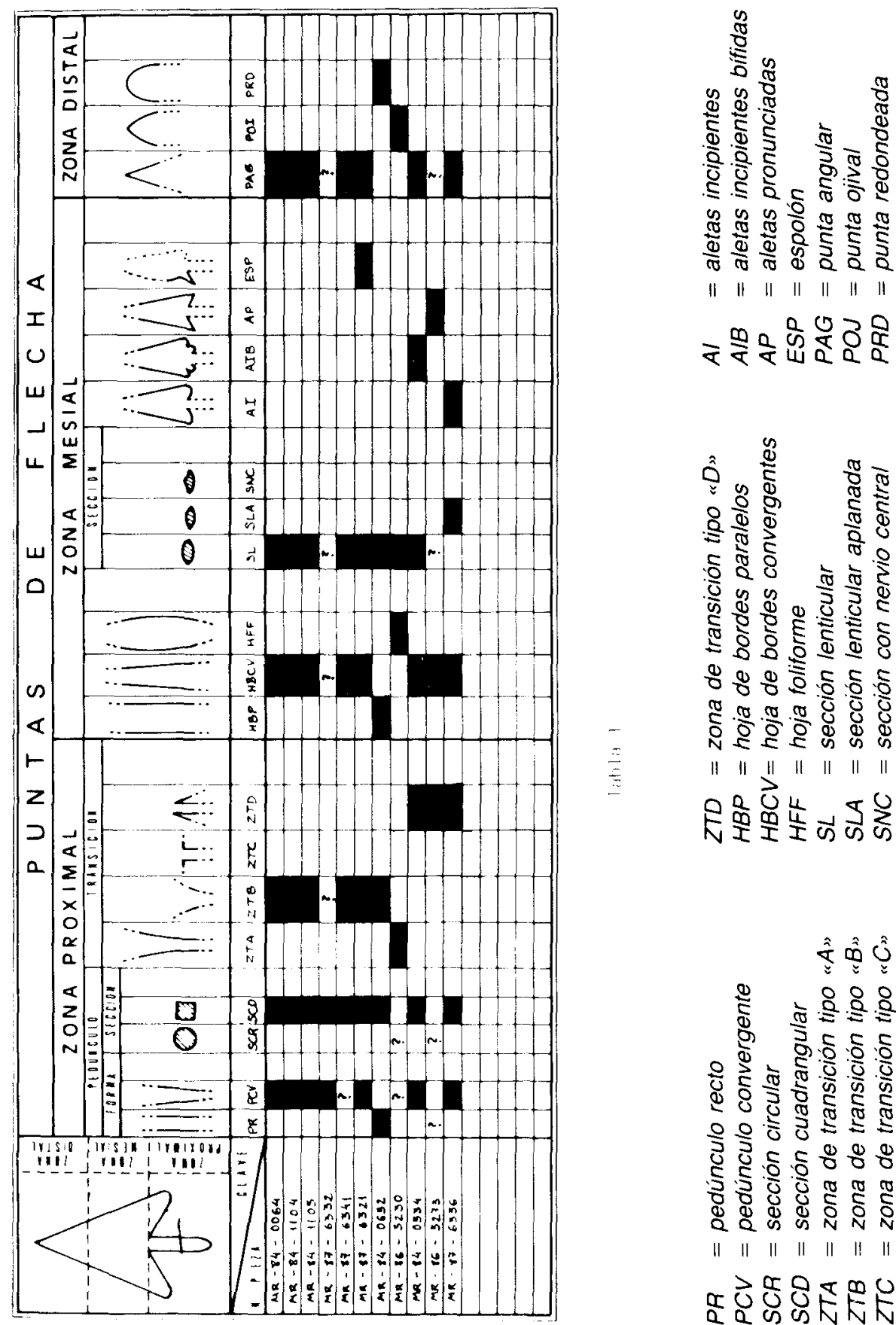

₹ิ $\hat{\hat{O}}_{\Sigma}^{\hat{j}}$

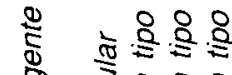

कृ 월

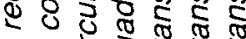
은응 త్ 3.

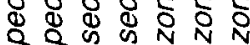
|| || || || || || || a 
b) Zona mesial: forma de la hoja

Hay un claro predominio de las puntas de hoja triangular, ya que de las 11 estudiadas, sólo una presenta los bordes paralelos y otra es de perfil foliforme. En cuanto a la sección trasversal de estas hojas, siempre que ha sido posible identificarla, debido a su estado de conservación, se puede observar un predominio absoluto de la sección lenticular y la aparición esporádica de una punta con la hoja ligeramente aplanada en la zona central de su cara superior y, en consecuencia, con los bordes ligeramente biselados (MR-87-6336).

c) Zona mesial: transición de la hoja al pedúnculo

Evidentemente, también esta parte de las puntas de flecha presenta una determinada forma en función de la de la hoja, asi como también en función de la presencia o ausencia de aletas. En este sentido se puede observar como en un solo caso, aquel en que la hoja es foliforme, la unión de ésta con el pedúnculo presenta un suave perfil divergente, mientras que en las puntas de hoja triangular se observan dos "situaciones": Ias tres piezas que tienen aletas claramente diferenciadas presentan esa zona de transición hoja-pedúnculo con perfil angular oblicuo hacia abajo, mientras que en otras seis puntas - por tanto, la mayoria-, dicho pertil es cóncavo, por lo que, al quedar muy marcada la diferenciación de la hoja y el pedúnculo, el extremo inferior de la misma presenta dos pseudoapéndices; este hecho ha dado lugar a que en otras ocasiones ambos lados de la zona inferior de la hoja se hayan identificado como pequeñas aletas, por lo que las puntas que nos ocupan responden a la denominación tradicional de "puntas de aletas incipientes o iniciadas".

Por úitimo cabe señalar que la punta núm. MR-87-6332 se halla en tan mal estado de conservación que nos es imposible identificar las caracteristicas de su hoja.

\section{d) Apéndices}

Sólo cuatro de estas 11 puntas de flecha presentan apéndices, tratándose de aletas cortas y pronunciadas en dos de ellas (MR-87-6336 y 
MR-86-5237, respectivamente), aletas biapuntadas en el caso de la pieza MR-84-0534, y un pequeño espolón lateral en el caso de la punta MR87-6321.

e) Zona proximal

Esta parte de las puntas está constituida por el pedúnculo, de perfil convergente y sección cuadrangular en la mayoría de los casos, y de perfil de lados paralelos y sección cuadrangular sólo en uno. Cabe señalar también que tal vez en el caso de las piezas MR-86-5230 y MR86-5273 la sección de sus pedúnculos fuese circular, pero su estado de conservación es muy deficiente y el grado de corrosión en que se encuentran impide apreciarlo con claridad.

\section{B. Dimensiones}

Refiriéndonos ahora solamente a las puntas que se encuentran completas, se puede observar que la longitud total de la más larga es de $85 \mathrm{~mm}$, y la de la más corta de $43 \mathrm{~mm}$, siendo la longitud media de este conjunto de piezas de $70 \mathrm{~mm}$, dato este que coincide con lo observado en otra ocasión ${ }^{2}$ para las puntas de flecha pedunculadas de la submeseta Sur.

En cuanto a la anchura máxima de estas puntas, es sensiblemente uniforme, oscilando entre los 11 y los $13 \mathrm{~mm}$ salvo en dos casos en que esa anchura alcanza los $15 \mathrm{~mm}$ (MR-87-6336) y hasta los $21 \mathrm{~mm}$ (MR86-5273).

Por último y respecto a la longitud del pedúnculo, consideramos, en función de la relación con la longitud de la hoja, como puntas de pedúnculo "corto" aquellas en las que la longitud de ésta es mayor que la del pedúnculo, y puntas de pedundulo "largo" aquellas en las que la longitud del mismo es igual o mayor que la de la hoja. Así podemos observar como en los casos en que ambas dimensiones se conocen, dado el

\footnotetext{
?Hernando Grande, A.: "Materiales Metálicos de la Edad del Bronce en la Meseta. Las armas". Tesis Doctora! leida en la U.N.E.D. (inédita).
} 
estado de conservación de las piezas, hay un predominio de las puntas de pedúnculo corto, coincidiendo dos de los tres casos en que aparece un pedúnculo largo con el hecho de que se trata de puntas con aletas.

\section{C. Peso}

No resulta muy indicativo este dato en el caso que nos ocupa puesto que, aunque a priori pensamos que deberia existir una relación entre la tipologia de las puntas de flecha y su peso, sin embargo las piezas presentan diferentes niveles de corrosión, lo que hace que sus pesos, además de no ser actualmente los originales, no sean comparables entre si.

No obstante cabe señalar una cierta relación, lógica por otra parte, entre el peso y la longitud de estas puntas, ya que la más corta y la más larga son las que menos y mas pesan, respectivamente.

\section{D. Composición}

No es nuestra intención hacer aqui un análisis exhaustivo de la composición del metal con que fueron fabricadas las puntas de flecha que estudiamos, pues ello nos llevaria indefectiblemente a establecer comparaciones con otros conjuntos de materiales del Bronce de la Mancha, $e$ incluso a plantear interrogantes acerca del origen de dicho metal, y otras muchas, todo lo cual excede los límites de este trabajo.

Pero ello no implica que no hagamos un somero comentario sobre algo que resulta evidente, y es, por una parte el hecho de que se trata de piezas de cobre y no de bronce, pues las cantidades de Sn detectadas son ínfimas, y por otra que, sin embargo, las cantidades de arsénico son muy variables, oscilando entre el 0,228 por 100 de la pieza MR-841104 y el 3.558 por 100 de la punta MR-87-6332. Es más, así como no hay una relación clara y evidente entre la morfología de las piezas y su composición metalográfrica, puesto que entre éstas que tienen una mayor proporción de As se encuentran una de hoja triangular, otra de hoja triangular con espolón y dos con aletas, sin embargo estas puntas de cobre arsenical se hallaron a mayor profundidad que las que apenas 
tienen As en su composición, lo cual nos lleva a plantear la posibilidad de que se trate de "lotes" de piezas fabricadas en momentos diferentes; de cualquier forma esto es algo que solo podemos señalar aquí a modo de hipótesis, puesto que solo podremos tener la constancia absoluta de ello hasta que contemos con los resultados del análisis estratigráfico, actualmente en curso de realización y estudio.

\section{Puñales}

Aunque son sólo tres los puñales encontrados hasta el momento en la Motilla de Santa María del Retamar, y los tres son morfológicamente parecidos, sin embargo no son totalmente iguales (tabla 2), como exponemos seguidamente.

\section{A. Morfologia}

a) Extremo distal: como en el caso de las puntas de flecha, la forma de la hoja condiciona la del extremos distal de la pieza, por lo cual mientras los dos puñales de hoja triangular presentan su punta angulosa, sin embargo la pieza número MR-87-5234, de hoja foliácea, consecuentemente presenta una punta de forma ojival.

b) Zona Mesial: En consecuencia con lo que acabamos de señalar, la forma de la hoja de dos de estos puñales es triangular, de filos rectos y "corta", ya que la longitud de las piezas no llega al doble de su anchura máxima, mientras que el tercero presenta una hoja foliforme de filos convexos, cuya proporción longitud-anchura máxima permite identificarla como "muy larga", Por lo que respecta a la forma de la sección de estas hojas, en dos de ellas es oval, mientras que en la tercera (MR-85-3506) es lenticular.

c) Zona Proximal. La zona correspondiente a la empuñadura de estos puñales es muy semejante en el caso de los dos triangulares, presentando una forma ligeramente convexa, y dos orificios para los correspondientes remaches, situados "en línea", pero en los bordes laterales de la misma. Sin embargo el puñal de hoja foliforme presenta una empunadura cuadrangular con tres orificios para remaches dispuestos 


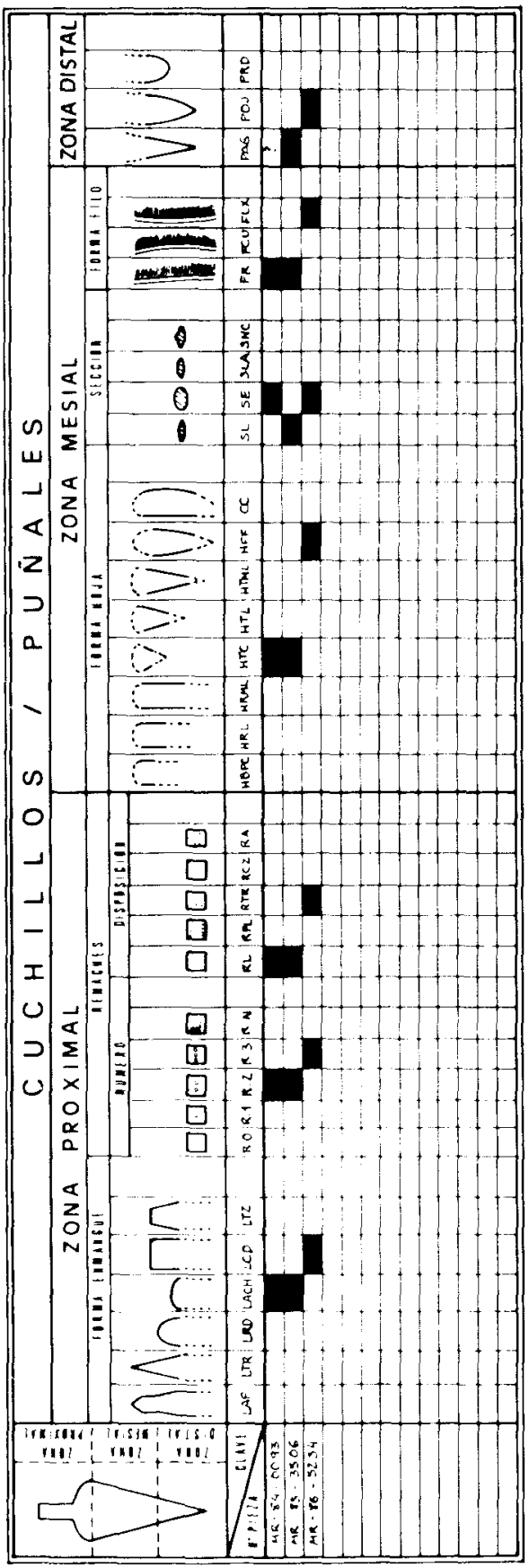

5.

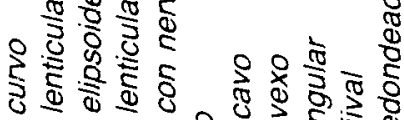

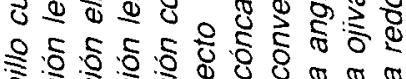

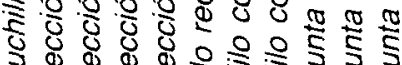

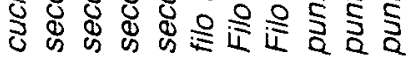
\| $\|$ || || || || || || || || $\mid$

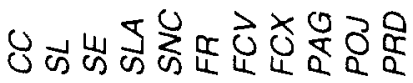

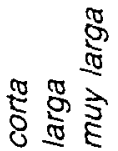

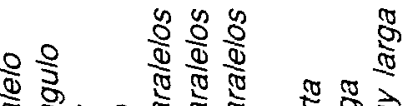

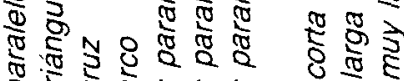

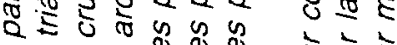

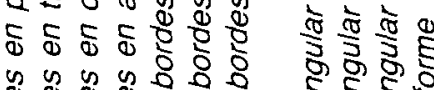

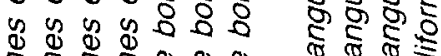

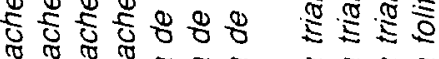

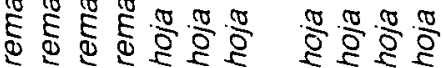
|| || || || || || || || || || ||

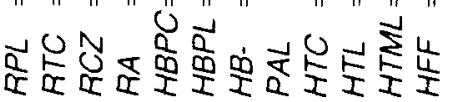

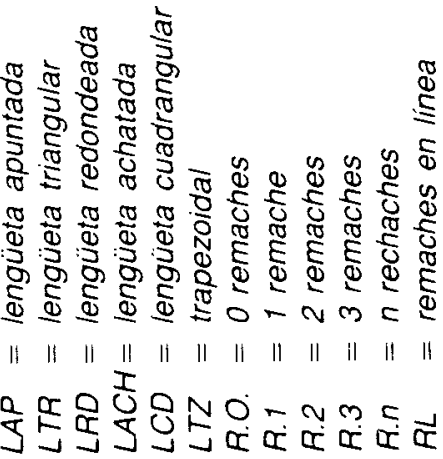


formando un triángulo, dos de ellos enfrentados en los bordes laterales y el tercero en la parte central del borde superior.

\section{B. Dimensiones}

Por 10 que respecta a las dimensiones de estas tres piezas, cabe señalar que mientras la longitud de los dos puñales "cortos" roza los cinco cms y su anchura no alcanza los tres cms, sin embargo el puñal foliforme tiene una longitud de $15.5 \mathrm{~cm}$ y una anchura máxima de 2.5 $\mathrm{cms}$, lo que, como apuntábamos antes nos lleva a considerarlo como «muy largo».

2. C. Peso

Aún cuando, como en el caso de las puntas de flecha, este dato no resulta demasiado indicativo, dado el diferente estado de conservación de las piezas, sin embargo cabe destacar el peso del puñal mas grande (MR-85-5243), que es de $90.630 \mathrm{~g}$.

\section{D. Composición}

También como en el caso de las puntas de flecha, el análisis de los componentes del metal con que fueron fabricados estos puñales indica, por una parte que se trata de piezas de cobre, y por otra que el contenido en arsénico es diferente de unas piezas a otras, pues mientras en el puñal triangular analizado su presencia es mínima, sin embargo en el puñal de mayores dimensiones ese elemento alcanza valores muy próximos a los que vimos en algunas puntas de flecha. En principio podríamos pensar que aqui si hay una relación directa entre las dimensiones de la pieza y su composición metálica, pues un puñal «muy largo" lógicamente requiere una hoja de mayor consistencia que las piezas cortas; no obstante, y dado que también en esta ocasión se da la coincidencia de que el puñal a que nos referimos se halló a mayor profundidad que los otros 
dos $-\mathrm{y}$ recordemos que uno de ellos se recogió en superficie-, debemos dejar abierta la posibilidad de que esas diferencias puedan tener otra explicación que esperamos obtener del estudio estratigráfico del yacimiento.

\section{CLASIFICACIÓN TIPOLÓGICA}

A la vista de las características de las piezas analizadas, podemos concluir que, de acuerdo con el sistema de clasificación propuesto por una de nosotras ${ }^{2}$ para las armas metálicas de La Meseta, las de la Motilla de Santa Maria del Retamar responden a los siguientes tipos:

a) Puntas de Flecha. Teniendo en cuenta que, pese a las diferencias observadas, tratamos de establecer la existencia de unos tipos "genéricos" que englobasen las características mas comunes y tambien que de algún modo permitiesen asumir las clasificaciones tradicionales, en nuestra tipología estas puntas de flecha responden a los tipos:

- PF.III.B.1 (puntas triangulares de aletas incipientes): piezas núms. $1,3,4,5,6,8,9$ y 11 .

- PF.III.B.2 (puntas triangulares de aletas pronunciadas): piezas núms. 2,7 y 10 .

b) Puñales. Por las mismas razones, es decir, en un intento de agrupar las piezas en unos tipos identificados en función de caracteristicas muy generales y cuya denominación resultase totalmente discordante con la tradicional, los puñales que presentan las características de los que estudiamos responden a nuestro tipo P.II.A.1 (punales de remaches planos y triangulares).

\section{SITUACIÓN EN EL YACIMIENTO Y CONTEXTO}

La Motilla de Santa María del Retamar es uno más de los asentamientos que, por sus especiales características de emplazamiento, morfologia, contexto material, etc., han permitido identificar una facies 
especifica del gran Complejo Cultural que ocupó una gran parte de la Submeseta Sur de la Península lbérica durante el II milenio a.C, el Bronce de la Mancha ${ }^{3}$, si bien la existencia real de dicha facies como tal, y no como verdadera cultura, no siempre ha sido aceptada por todos los investigadores de la Protohistoria manchega y ha sido discutida por otros, aunque hoy para muchos, entre los que por supuesto nos encontramos, es evidente.

A la localización y caracteristicas del yacimiento ya nos referimos en otro trabajo ${ }^{4}$, por lo que no insistiremos aqui en ello, pero sí nos detendremos sin embargo en lo referente a la situación de las armas en la motilla.

En efecto, no deja de ser ciertamente interesante el hecho de que todas las puntas de flecha, asi como dos de los puñales localizados -el tercero (MR-85-3506) se recogió en superficie, como ya señalamos-, se encontrasen en un reducido espacio del cuadrante $S E$ del yacimiento, en el denominado "Corte A"; concretamente ese espacio es el comprendido entre los dos recintos de fortificación que rodean a la torre central, y que se encontraba ocupado por los restos de las estructuras construidas adosadas a la cara interna del segundo de dichos recintos, de tal modo que estas piezas metálicas se hallaron incrustadas en los escombros de tapial que, junto con los restos de las cubiertas de las habitaciones correspondientes, rellenaban ese espacio intramuros.

En este sentido hemos de señalar, por una parte, que esa zona del yacimiento es también en la que se ha excavado hasta una mayor profundidad (algo mas de $4 \mathrm{~m}$ desde el punto más alto de la motilla), por lo que hemos de dejar abierta la posibilidad de cuando la excavación alcance cotas semejantes en otras zonas del yacimiento, el panorama relativo a la situación de los hallazgos cambie, pero por otra parte hemos de señalar también que no creemos que la concentración de armas en el espacio citado se deba a la casualidad, como tampoco que se trate de un depósito intencionado, ya que las piezas aparecieron próximas, pero no juntas.

${ }^{3}$ Nieto Gallo, G. y Sanchez Meseguer, J.: “Bases para la sistematización del estudio de la Edad del Bronce de la Mancha". Actas del I Congreso de Historia de Castilla-La Mancha, t. II. Junta de Comunidades de Castilla - La Mancha. Toledo 1988, págs. 221 y ss.

" Colmenarejo, R., Galan, C., Martinez, J. y Sanchez Meseguer, J.: "La 'motilla' de Santa Maria del Retamar (Argamasilla de Alba, Ciudad Real)", Oretum, III. Museo de Ciudad Real. Ciudad Real 1988, pág. 5 y ss. 
Por lo que se refiere a los puñales, y excepción hecha del hallado en superficie, aparecieron revueltos con esos mismos escombros, pero no incrustados en ellos, de tal modo que todo parece indicar que, mientras éstos bien pudieron pertenecer a los ocupantes de la motilla, en cuyo caso nada nos impide pensar que formasen parte de su equipo material y que, por tanto, formaban parte del contexto global del yacimiento, sin embargo, por lo que respecta a las puntas de flecha, y ante el hecho de que se hallaran incrustadas en los restos de las paredes de tapial y muchas de ellas arqueadas o con sus puntas dobladas como consecuencia de haber sido lanzadas contra las estructuras construidas entre los dos recintos de fortificación, parece evidente que no tendría mucho sentido pensar que ello se debiese a la acción de los propios habitantes del asentamiento que hubiesen disparado sus puntas de flecha contra su propio lugar de habitación. De esto se deduce que si estas armas fueron lanzadas desde fuera por "otros", no podemos asociarlas al contexto global del yacimiento, pues no formaron parte del equipo material de sus ocupantes, todo lo cual apoya a su vez la hipótesis que ya planteamos en otro momento ${ }^{4}$ respecto a la aloctonia de las mismas.

Una vez formuladas las respuestas para las preguntas sobre como son, dónde se encontraban y qué contexto acompañaba a las armas que estudiamos, veamos ahora las circunstancias que rodean a este tipo de objetos en otros yacimientos del Bronce de la Mancha.

En este sentido hemos de señalar que solo podemos utilizar en este caso como término de comparación el yacimiento del Cerro de La Encantada, dado que, pese a que en otros yacimientos de este complejo cultural se han realizado varias campañas de excavación, como es el caso de la motilla de El Azuer, en Ciudad Real ${ }^{5}$ o de El Acequión, en Albacete ${ }^{6}$, sin embargo no contamos con la suficiente documentación publicada como para analizar la situación al mismo nivel.

Como señalamos recientemente ${ }^{2}$, el Cerro de La Encantada y la Motilla de Santa María del Retamar son, hasta el momento, los dos ya-

"Najera, T. y otros: "La motilla de El Azuer (Daimiel, Ciudad Real)». N.A.H., VI. Ministerio de Cultura. Madrid 1979, págs. 21 y ss.; "La Edad del Bronce en La Mancha. Las motillas de El Azuer y Los Paiacios", Cuadernos de Prehistoria de la Universidad de Granada, 2. Granada 1977, págs. 251 y ss.; "Excavaciones en las Motillas de El Azuer y Los Palacios", Actas del XIV C.A.N., Zaragoza 1977, págs. 503 y ss.

- Fernandez-Miranda, M., Fernandez - Posse, M. D. y Martin Morales, C.: "Caracterización de la Edad del Bronce en La Mancha. Algunas proposiciones para su estudio", Espacio, Tiempo y Forma, Serie 1. Prehistoria Homenaje al Profesor Eduardo Ripoll Perelló. UNED, Madrid 1988, pags. 293 y ss. 
cimientos del Bronce de la Mancha que mayor número de armas metálicas han proporcionado, pero tanto las características del conjunto de ellas, en cada caso, como el contexto en que se hallaron son sensiblemente diferentes. Así, mientras en la motilla que nos ocupa hay un claro predominio de las puntas de flecha y su presencia parece deberse, como acabamos de ver, a un ataque desde el exterior, sin embargo en el Cerro de la Encantada, el mejor y más intensamente conocido representante de la facies "Castellones" del mismo Complejo Cultural, no solo las armas predominantes son los punales, sino que tambien el contexto, o mejor dicho, los contextos en que aparecen dichas armas son sensiblemente diferentes al de la motilla, puesto que tanto puñales como puntas de flecha se hallan bien en niveles con restos de habitación, bien formando parte de los ajuares funerarios de sepulturas de diferentes tipos, entre las que se encuentran desde inhumaciones en grietas hasta los denominados "Complejos", edificios de carácter religioso asociados, de diversas formas, a sepulturas sensiblemente diferenciadas por sus características en el conjunto funerario del yacimiento?

En resumen pues, mientras en la motilla de Santa Maria del Retamar la mayor parte de las armas localizadas hasta el momento, concretamente las puntas de flecha, no debieron formar parte del equipo material de uso habitual de sus habitantes, y los puñales bien pudieron haberlo hecho, aunque, al menos de momento, no tenemos evidencias de que estos fuesen de fabricación local, sin embargo en el Cerro de la Encantada todo indica que ambos tipos de armas formaron parte de la panoplia y utilillaje de algunos individuos, y parece evidente que tuvieron un significado muy especial, en tanto que, cuando aparecen como objetos votivos, están asociados a sepulturas "especiales" ya sea por la riqueza de su ajuar (brazalete de plata), por sus propias características (sepultura de fosa revestida de mampostería, con inhumación doble), y/o por su asociación a algunos de los "Complejos Funerarios" y todo ello sin olvidar que el Cerro de La Encantada ha proporcionado no solo estructura y materiales relacionados con la producción metalúrgica (hornos de fundición, escoria, etc.), sino también un molde para la fundición de puntas de flecha procedente concretamente del "Complejo 7".

\footnotetext{
Romero Salas, H. y Sanchez Meseguer, J: "La faciés necropolis del Cerro de La Encantada. Aproximación a su estratigrafía", Actas del / Congreso de Historia de CastillaLa Mancha, t. III. Junta de comunidades de Castilla-La Mancha. Toledo 1988, págs. 139 y ss.; Romero, H., Sanz del Cerro, E. y Sanchez Meseguer, J.: "La Encantada: Bronce de La Mancha o Bronce Argárico?", Idem, págs. 119 y ss.; Romero Salas, H. y SANCHEz Meseguer, J.: "La facies necrópolis del Cerro de La Encantada", Oretum, IV. Museo de Ciudad Real. Ciudad Real (en prensa).
} 


\section{CONCLUSIONES}

Este análisis de las armas metálicas de la motilla de Santa Maria del Retamar, de sus caracteristicas, las del yacimiento y las circunstancias que las rodeaban en el momento de su hallazgo, nos permite plantear, a modo de conclusión, una hipótesis, que tal vez futuras investigaciones en el yacimiento permitan confirmar, respecto al significado de la presencia de las mismas.

Ciertamente, estos puñales y puntas de flechas responden claramente a los tipos constatados en otros yacimientos del Bronce de la Mancha, y tanto correspondientes a la facies "motillas" como a las facies "morras" y "Castellones" de este Complejo Cultural, habiéndose constatado la presencia de puntas de flecha foliformes en el Cerro de La Encantada (Ciudad Real) y el Acequión (Albacete), de puntas de pedúnculo $y$ aletas en esos dos yacimientos y otros como las motillas de Azuer y Los Palacios (Ciudad Real), el Cerro del Cuco (Cuenca) y el Castellón de Infantes (Ciudad Real) ${ }^{8}$, y de puñales de remaches en motillas como el Acequión ${ }^{5}$ o la Peñuela ${ }^{9}$, morras como el Cerro del Cuco y castellones como el Cerro de La Encantada ${ }^{2}$, siendo esporádica, al menos de momento, la presencia de puntas de flecha con «espolón» lateral constatada únicamente en la motilla que nos ocupa.

Ahora bien, si culturalmente habiando estas armas de metal están claramente inmersas en el contexto del Complejo Cultural a que corresponden todos estos yacimientos, sin embargo la comparación con aquel del que tenemos mas información, el Cerro de La Encantada, pone claramente de manifiesto que, al no hallarse siempre en idénticas circunstancias, la presencia de armas metálicas no puede interpretarse de la misma forma, como anteriormente señalamos, y, en el caso concreto de la motilla de Santa Maria del Retamar, pensamos que resulta evidente que si los puñales tal vez estaban allí porque alli los dejaron sus ocupan-

\footnotetext{
" Hernando Grande, A.: "Piezas metálicas de la Edad del Bronce en la Meseta: puntas de flecha triangulares con pedúnculo y aletas". Espacio. Tiempo y Forma, Serie I. Prehistoria. Homenaje al prołesor Eduardo Ripoll Perelló. UNED, Madrid 1988, págs. 311 y ss.

9 SANCHEZ JIMENEZ, J: "Excavaciones y trabajos arqueológicos en la provincia de Albacete de 1942 a 1946. Poblado y necrópolis de La Peñuela", Intormes y Memorias, 15, 1947. págs. 7 y ss.; "La cultura argárica en la provincia de Albacete. Notas para su estudio", Actas y Memorias de la Sociedad Española de Antropologia, Etnologia y Prehistoria (SEAEP), 23, 1948, pags. 96 y ss.
} 
tes, sin embargo las puntas de flecha llegaron hasta sus muros disparadas desde el exterior.

Este planteamiento deja abiertas evidentemente un buen número de interrogantes tales como:

¿quiénes fueron los autores del ataque?

¿desde donde dispararon?

¿por qué lo hicieron?

De momento, para el "quiénes" y el "por qué" no podemos ofrecer una respuesta, pues ésta depende de muchos aspectos del desarrollo del Bronce de la Mancha que, en el estado actual de las investigaciones aún no conocemos bien, pero si podemos intentar ver si es posible discernir desde dónde fueron lanzadas esas flechas contra la motilla.

Como ya señalamos, su situación en el yacimiento está muy localizada en un reducido espacio de cuadrante SE del mismo, lo que nos ha llevado a analizar la ubicación del yacimiento y la topografia y caracteristicas del terreno circundante (fig. 3), a fin de observar cual habria sido el lugar desde dónde se efectuó el ataque.

Asi hemos podido comprobar como es precisamente en dirección SE el punto hacia el cual la visibilidad desde la motilla es menor, es decir, que aun cuando evidentemente desde el yacimiento se ve la pequeña plataforma de afloramientos calizos que constituía la orilla del rio, sin embargo la visibilidad acaba prácticamente en ella, siendo mucho mas viable el control de un territorio más amplio en otras direcciones (fig. 4).

La representación topográfica del relieve del entorno de la motilla permite observar como esa pequeña plataforma acaba, en dirección al río. en un escarpe pequeño y de suave pendiente, pero que denota como el relieve de la orilla izquierda del Guadiana es en esta zona ligeramente más acusado que el de la orilla derecha. Utilizando una técnica de evidente valor para la Arqueología, la cartografía automática ${ }^{10}$, esas diferencias en el relieve y la relación entre éste y la motilla se aprecian mejor en el cariograma que presentamos (fig. 5), en el que, ademas de representar el yacimiento esquemáticamente y exagerando sus dimensiones reales a fin de destacar su presencia, se ha introducido una modificación respecto al paisaje actual: la representación de la zona inundada por las aguas del Guadiana cuando éste corría libremente, es decir, sin canali-

\footnotetext{
dita).

"Grandio de Fraga, E.: "Cartografia Automática en Arqueologia". Tesis Doctoral (iné-
} 


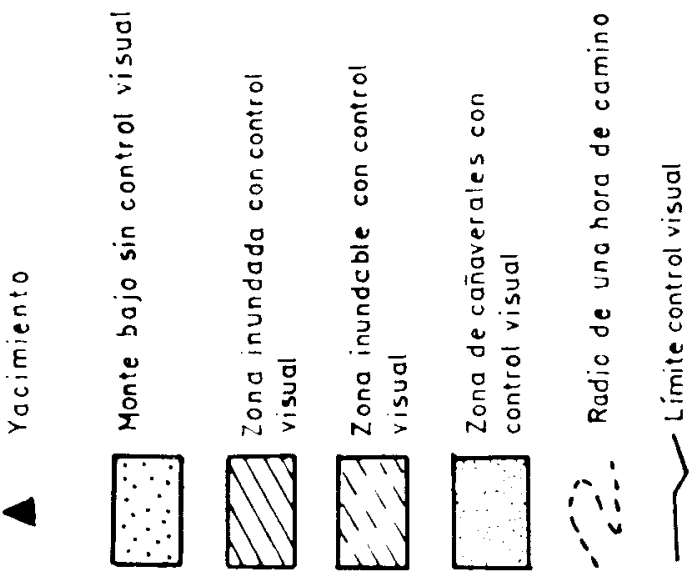




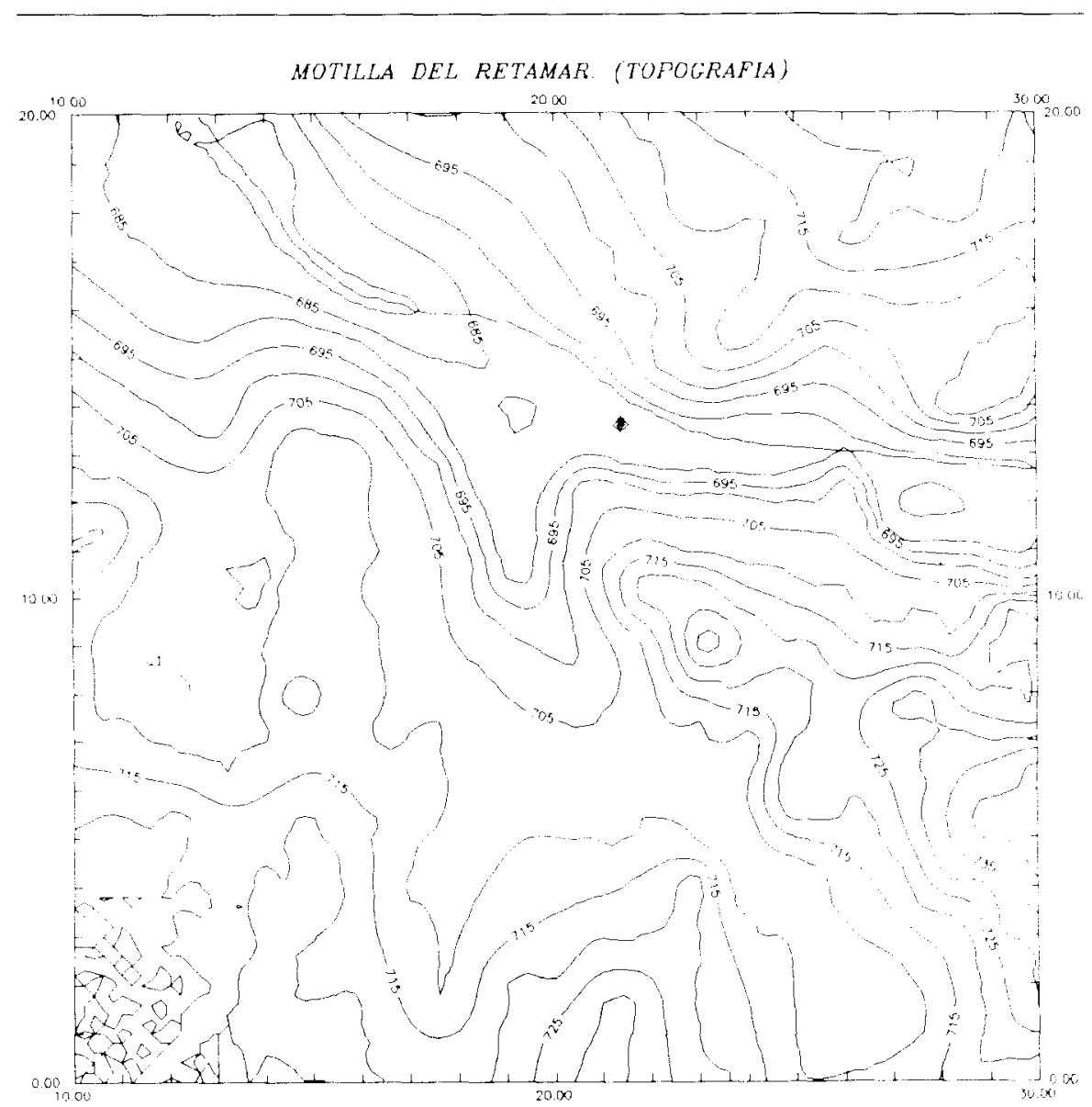

Fig. 4. Coordenadas UTM: $X=$ long. 496; $Y=$ lat. 4322 - 4328. Área comprendida: $2 \mathrm{Km}$ cuadrados.

zar, por el territorio, si bien es cierto que el nivel de altura del caudal del río se ha puesto en un óptimo de tres metros por encima de la cota anual del suelo cultivado al pie de la motilla - a la vista de los resultados obtenidos en la excavación del yacimiento- " todo ello permite observar

"Agradecemos ahora muy sinceramente la colaboración del Dr. D. Eduardo Grandio de Fraga en la elaboración de la representación gráfica de la topografía del yacimiento y su entorno; gracias también a D. Francisco Requena por su ayuda en esta parte del trabajo. 


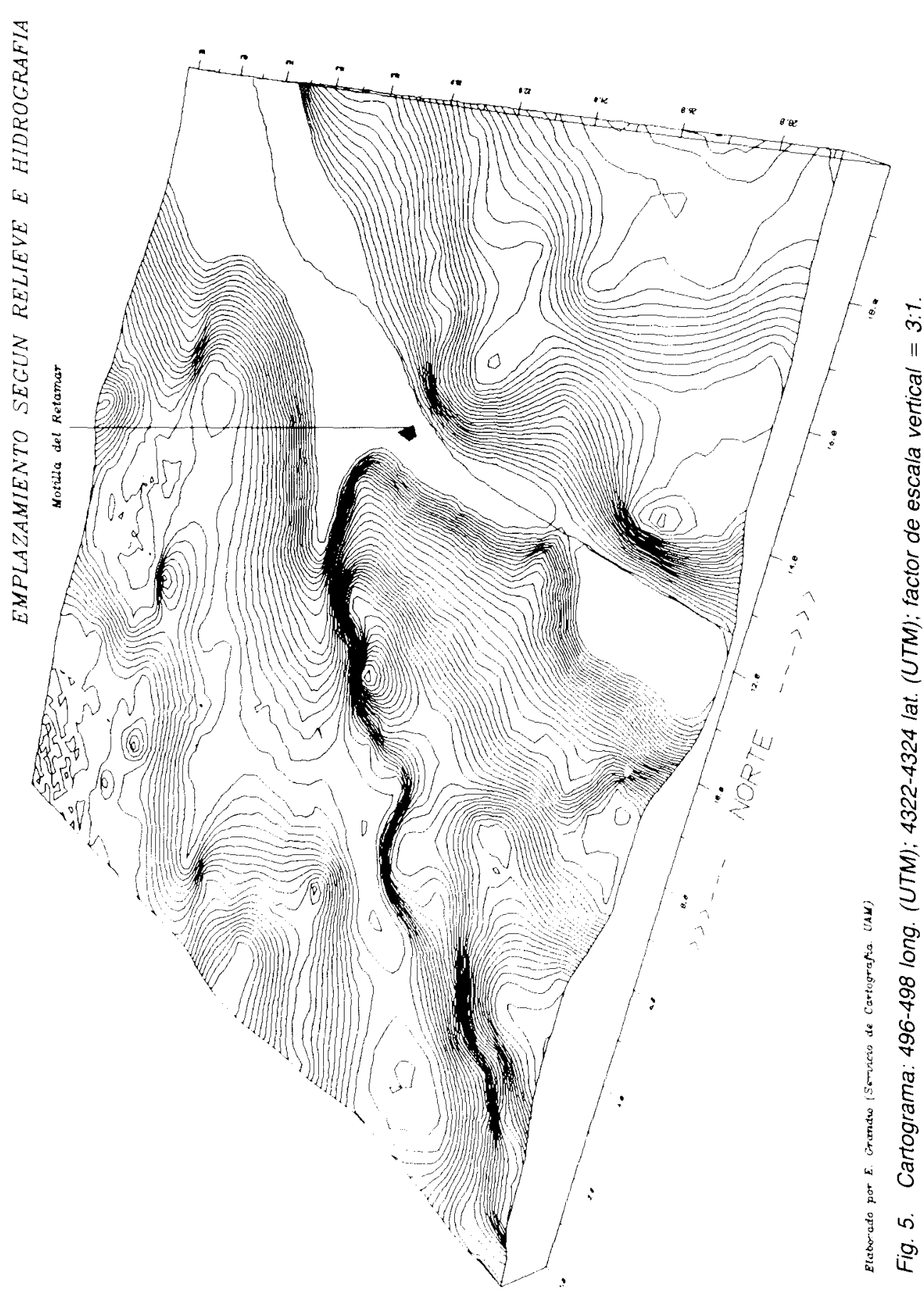


como esa pequeña plataforma de calizas es la utierra firme" más próxima al yacimiento, por lo que parece bastante claro que, desde el punto de vista de "los atacantes", este lugar sería el más apropiado desde el punto de vista estratégico para llevar a cabo el ataque.

Ahora bien, es cierto que esta reconstrucción del "escenario" en el que tuvo lugar uno de los episodios de la historia de la Motilla de Santa Maria del Retamar, plantea a su vez algunos problemas tales como los referentes al conocimiento de la visibilidad real desde la propia motilla o del lugar exacto desde el que fueron disparadas las flechas.

En este sentido, el cuestionar la visibilidad real del asentamiento en definitiva a lo que nos lleva es a plantearnos si se trató de un ataque directo, que pudiera haber sido claramente advertido por sus ocupantes, o más bien de una escaramuza por sorpresa, y ante ello hemos de tener en cuenta evidentemente que el panorama que se ofrecía a la vista de los habitantes de la motilla no era el que actualmente se contempla desde el punto más alto del yacimiento, ya que las aguas de un Guadiana no canalizado corrian lentamente por lo que hoy es la vega cultivada, a los pies de la motilla, y a ello hay que sumar el hecho de que la correspondiente vegetación ribereña permitía sin duda acercarse hasta la orilla del río, buscando lógicamente el punto de ésta más próximo al "objetivo", al amparo de los chopos, álamos, cañas, etc. que flanqueaban los bordes de su cauce. Esto quiere decir que mientras hoy los afloramientos calizos a que nos referiamos son perfectamente visibles desde la motilla, no debian serlo sin embargo, al menos no en la misma medida, en el II milenio a.C., ni siquiera aunque tengamos en cuenta que "el punto de mira" de sus ocupantes quedaría por encima de lo que hoy es la zona más alta del yacimiento, puesto que la altura de la superestructura de madera, tapial, etc. que debió soportar la torre central permitiria aumentar el campo de visibilidad.

En cuanto al punto desde el cual fueron disparadas las flechas, cabe plantear dos posibilidades: que lo fueran directamente desde la orilla del río, o bien que los asaltantes se acercasen hasta el pie de la motilla utilizando algún tipo de embarcación, necesariamente de fondo plano dada la escasa profundidad que debió alcanzar normalmente su cauce, y sorteando los juncos, cañas y demas tipos de vegetación propios de un lugar ocupado por las aguas pero dónde estas corren muy lentamente debido a la escasa pendiente del terreno. Pensamos que para conocer este extremo necesitariamos elementos de juicio suficiente para poder reconstruir la trayectoria que siguieron las flechas en cuestión, y ésto es algo que escapa a nuestras posibilidades puesto que, como ya señalamos, se encontraban entre los escombros de las estructuras que se de- 
rrumbaron muy posiblemente a consecuencia del incendio que siguió al ataque, lo que nos impide conocer la posición exacta en que quedaron clavadas en los tapiales tras su lanzamiento. No obstante, dada la distancia existente entre la motilla y la orilla del río, pensamos que es muy posible que el lanzamiento de las puntas de flecha se efectúase desde el pie del yacimiento.

Hemos expuesto hasta aquí nuestra interpretación de la presencia de armas metálicas en la motilla de Santa Maria del Retamar.

Si futuras campañas de excavación no permiten confirmar la hipótesis que hoy planteamos, habremos de buscar evidentemente otras explicaciones, pero si no es asi, es decir, si no encontramos pruebas de que las cosas sucediesen de otra manera, o al menos indicios de que así fuese, ello nos llevaria a preguntarnos si se trató de un hecho aislado o si, por el contrario, algo semejante sucedió también en otros asentamientos, lo que indefectiblemente nos llevaría a analizar la situación en otros yacimientos del Bronce Manchego en la medida en que ello fuese posible.

En cualquier caso, esperamos haber contribuido con estas líneas, desde las que queremos dejar constancia de nuestro agradecimiento al Dr. Sanchez Meseguer por su colaboración en la excavación y estudio de la motilla de Santa María del Retamar, no sólo a la publicación de las caracteristicas de algunos de los materiales de nuestro yacimiento, sino también al planteamiento de la reconstrución de la Protohistoria manchega como una etapa de su Historia con sus épocas de nacimiento, desarrollo y esplendor, pero también con sus conflictos y problemas ${ }^{12}$.

${ }^{12}$ Nuestro agradecimiento, asi mismo, a D. Elena Fernández, autora de los originales a tinta de las tablas y dibujos de las piezas metálicas que acompañan a este trabajo. 